\title{
Stabilization of Near-Infrared Fluorescent Proteins by Packaging in Virus-like Particles
}

Soumen Das, ${ }^{1}$ Liangjun Zhao, ${ }^{1}$ Stephen N. Crooke, ${ }^{1}$ Lily Tran, ${ }^{2}$ Sonia Bhattacharya, ${ }^{1}$ Eric A.

$$
\text { Gaucher, }{ }^{2} \text { and M.G. Finn }{ }^{1,3 *}
$$

${ }^{1}$ School of Chemistry and Biochemistry, Georgia Institute of Technology, 901 Atlantic Drive, Atlanta, GA, 30306, USA

${ }^{2}$ Department of Biology, Georgia State University, Atlanta, GA 30303, USA

${ }^{3}$ School of Biological Sciences, Georgia Institute of Technology, 901 Atlantic Drive, Atlanta, GA, 30306, USA

* correspondence: M.G. Finn, e-mail: mgfinn@gatech.edu

\section{Supporting Information}
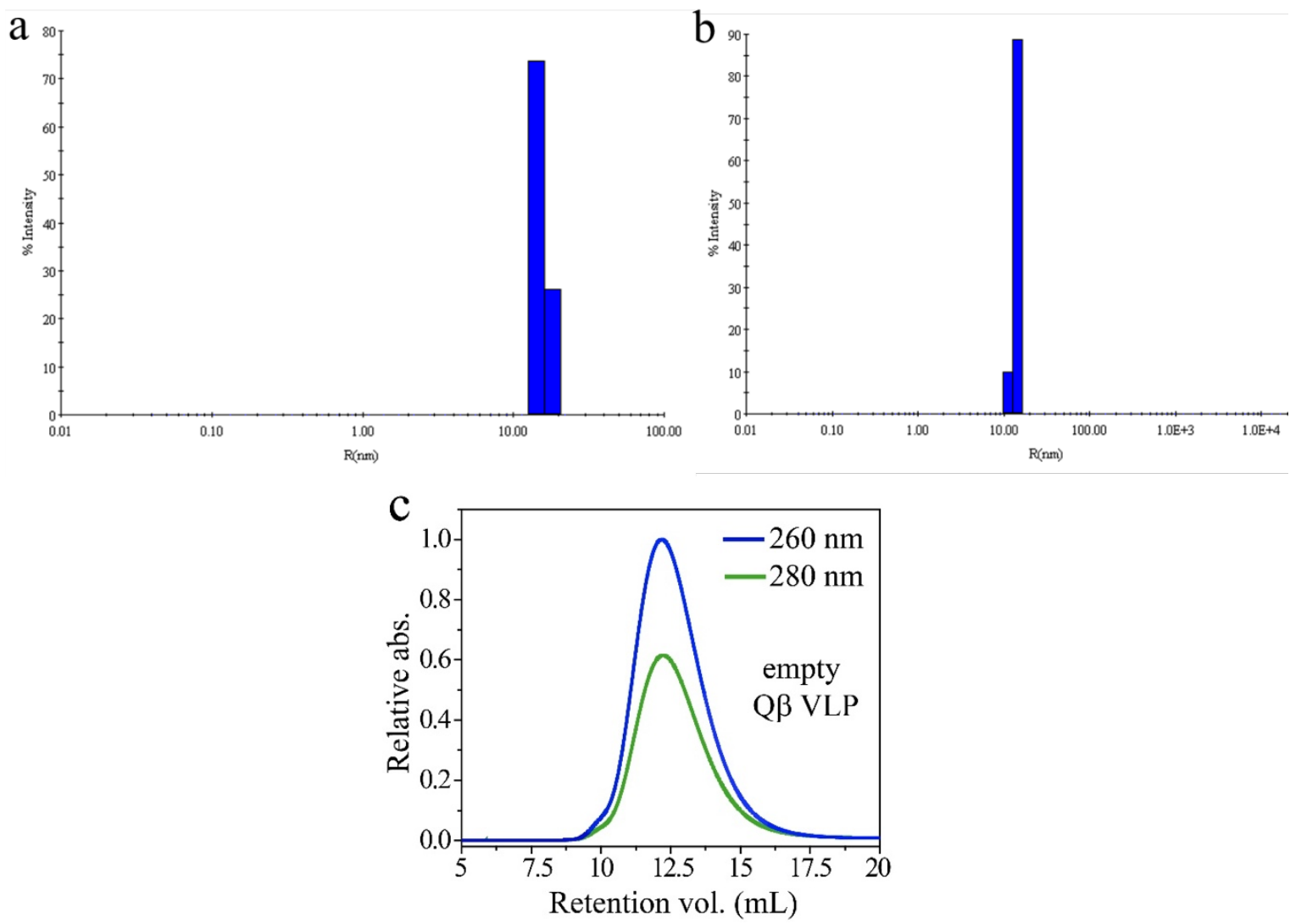

Figure S1. Dynamic light scattering (DLS) of (a) Q $\beta @$ iRFP720 and (b) Q $\beta @$ mIFP ${ }_{9}$ particles and (c) Size-exclusion FPLC of empty Q $\beta$ VLPs. No absorption at 680-700 nm was observed. 

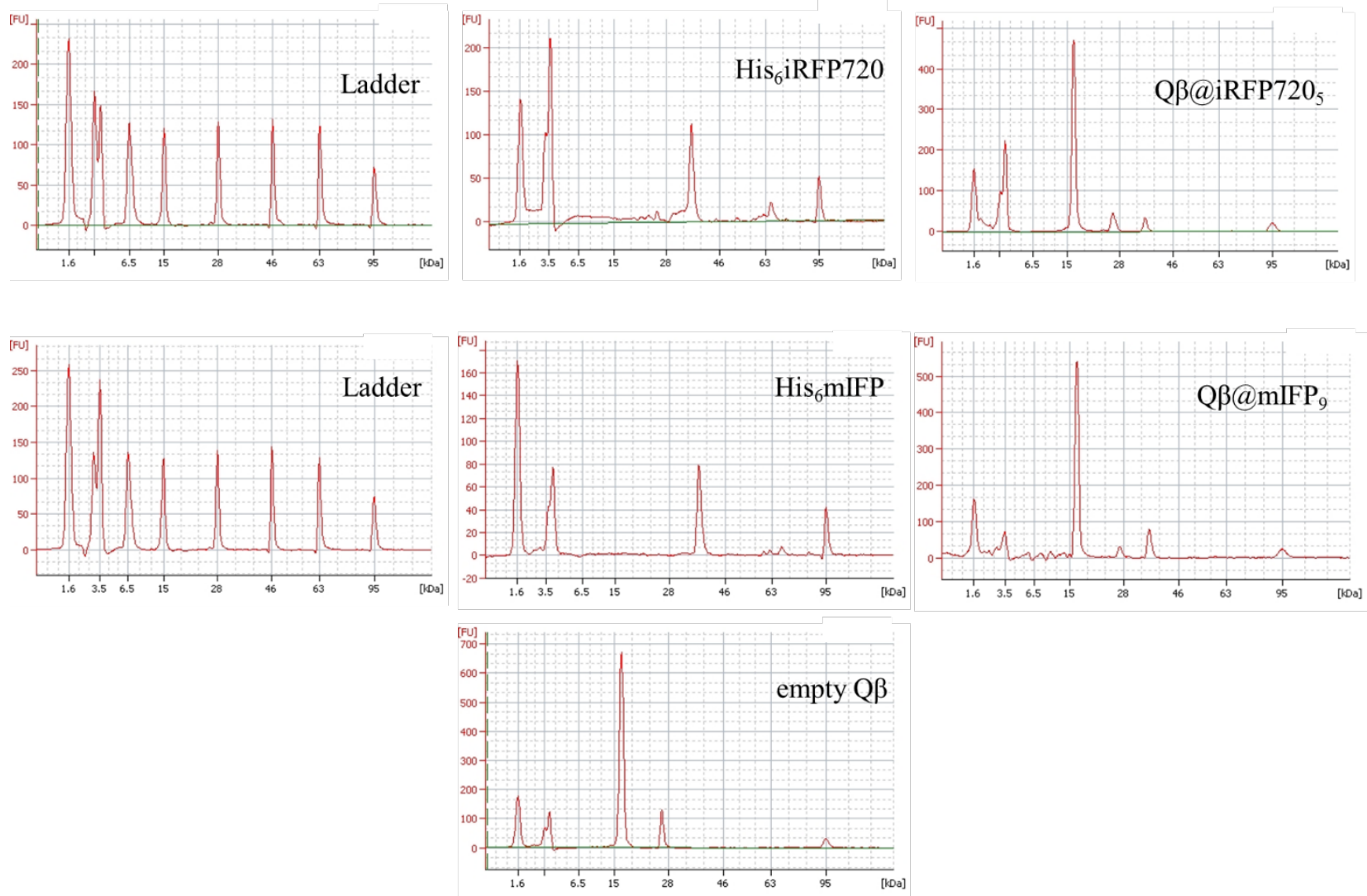

Figure S2. Electropherogram plots of the corresponding gel images presented in Figure 2. These plots are used to calculate average number of NIR FPs present in Q $\beta$ VLP.

Figure S3. Absorption spectra of biliverdin and Q $\beta @$ iRFP720.

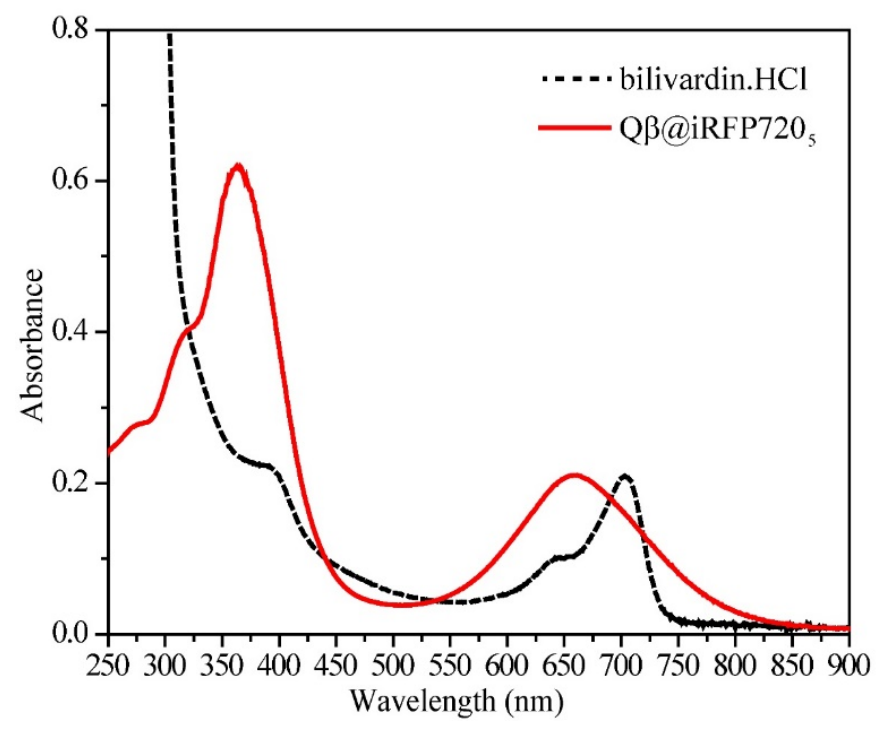



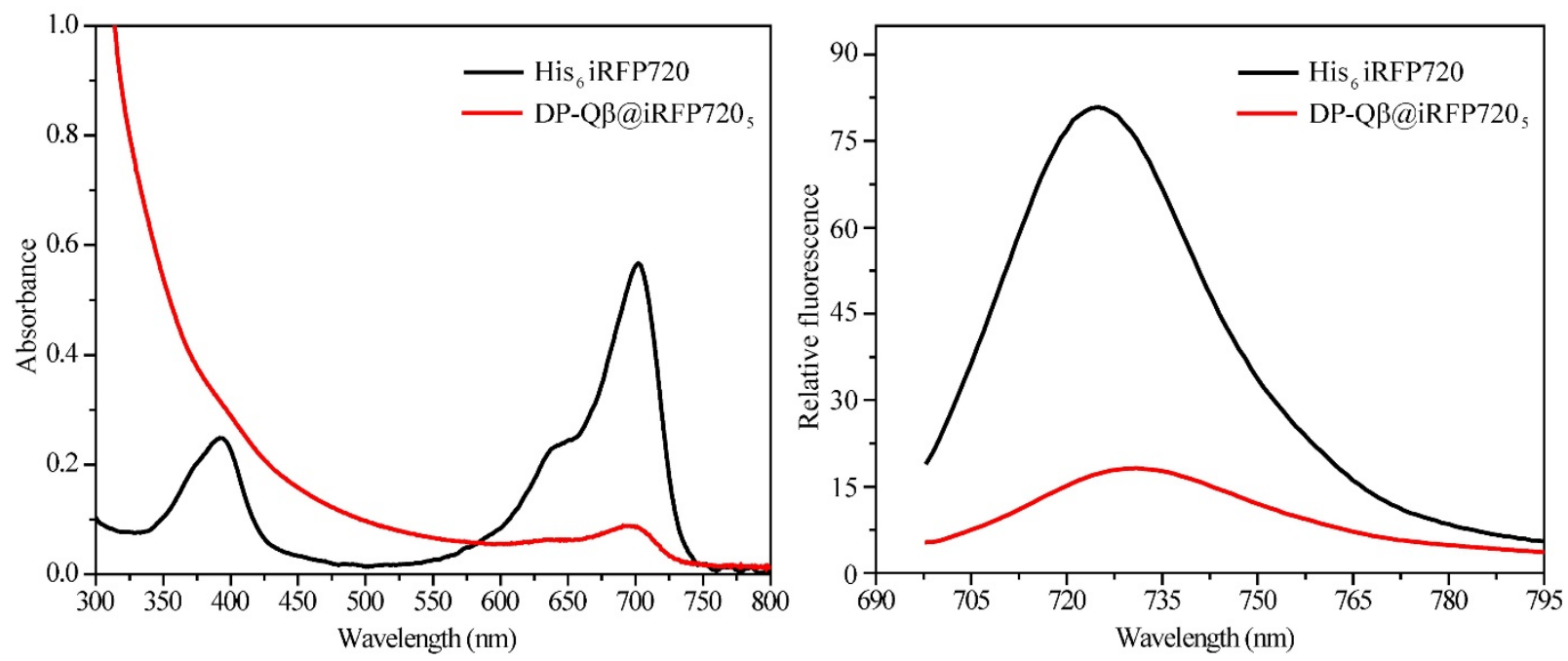

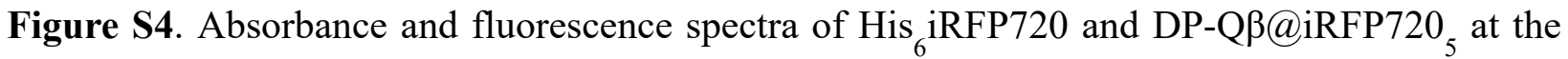
same concentration of iRFP720.
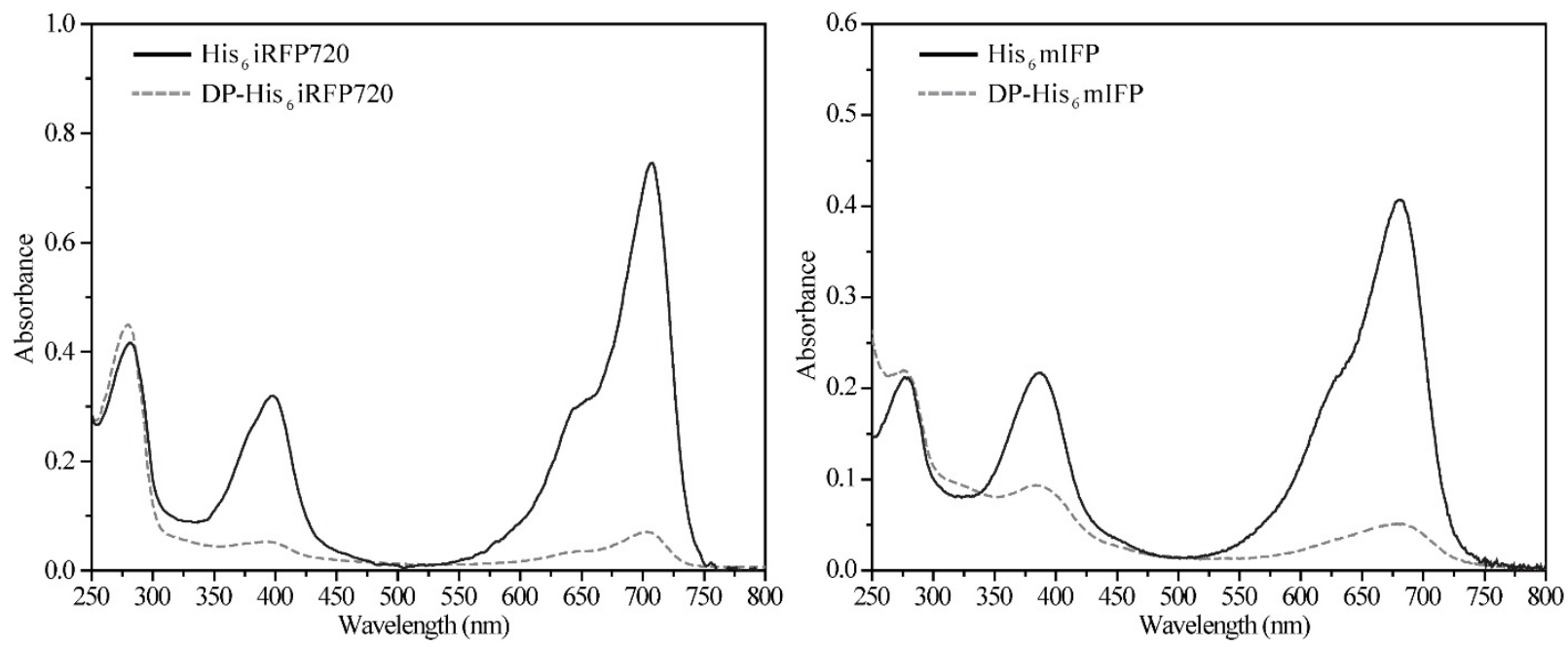

Figure S5. Free NIR FPs expressed in presence of HO and direct BV exposure, respectively. 

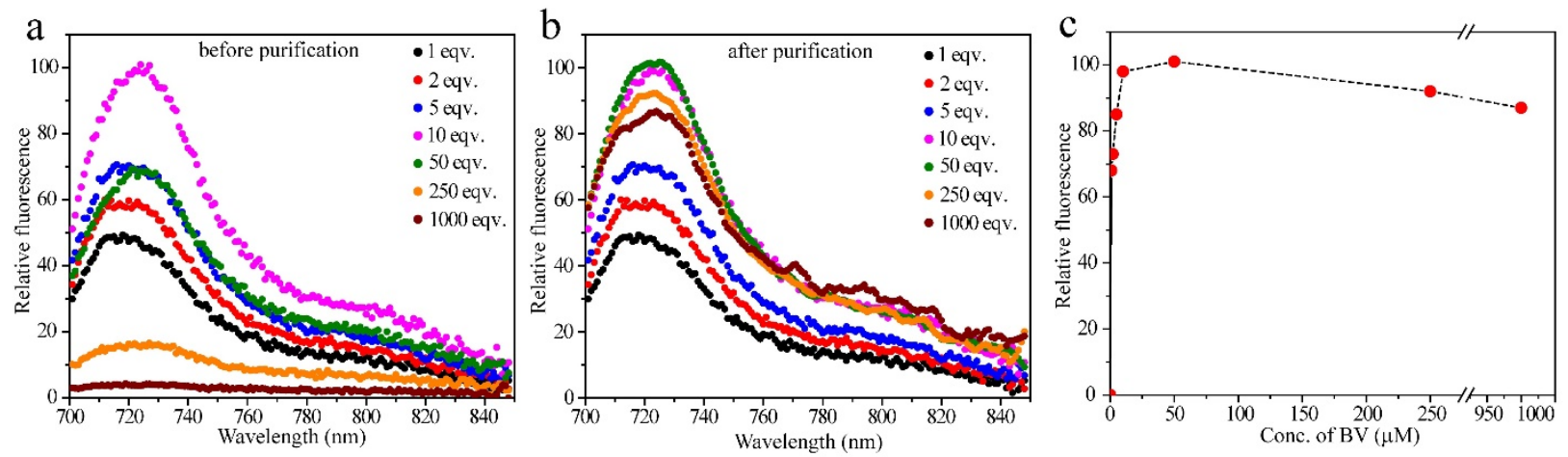

Figure S6. Incubation of freshly prepared Q $\beta @$ apo-iRFP720 $5(1 \mu \mathrm{M}$ of apo-iRFP720) with different amounts of BV: (a) fluorescence spectra of the samples before purification; (b) after purification; (c) plot of normalized fluorescence against concentration of biliverdin. All data normalized to the protein absorption at $280 \mathrm{~nm}$.

Figure S7. Q $\beta @$ apo-iRFP720 5 treated with $\mathrm{BV}$ at 1:10 ratio (apo-iRFP720:BV) in absence and in presence of DTT $(1 \mathrm{mM})$ respectively.
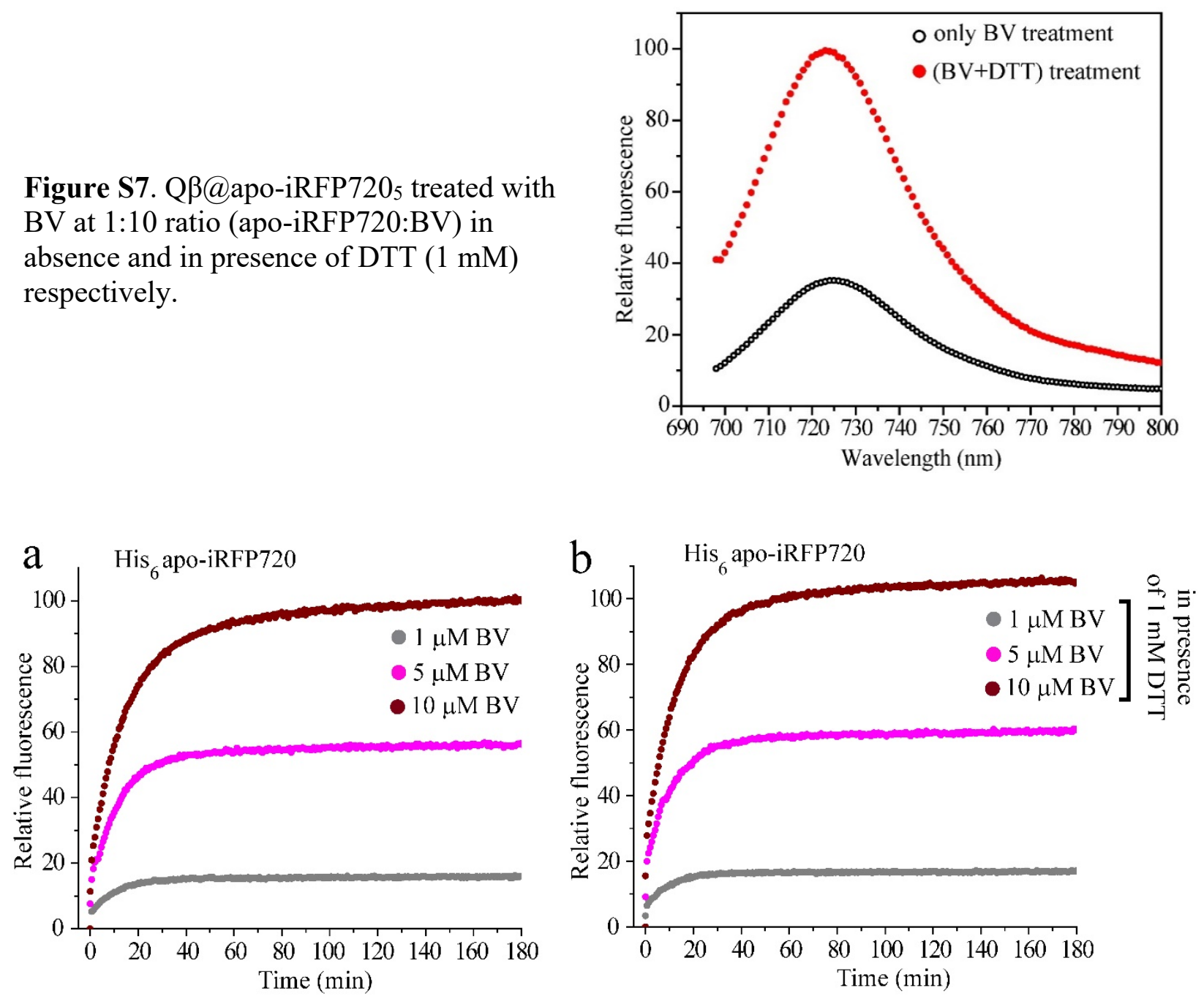

Figure S8. Maturation kinetics of apo-protein in presence of different concentrations of BV: (a) His 6 apo-iRFP720 and (c) His 6 apo-iRFP720 in presence of 1 mM DTT. 

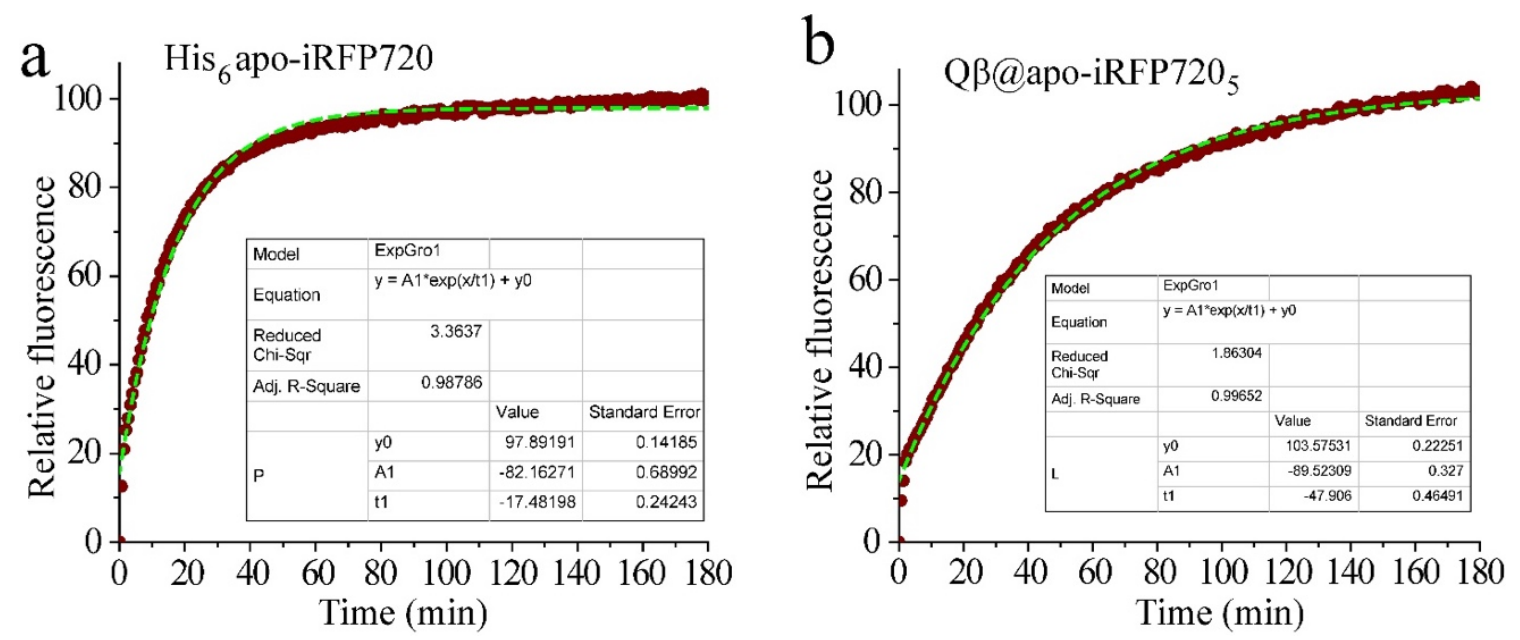

Figure S9. Calculation of maturation half-time of apo-protein in presence of 10 equivalent of BV and 1 mM DTT: (a) His 6 apo-iRFP720 and (c) His6apo-iRFP720. To calculate maturation halftime, the data were fitted into $1^{\text {st }}$ order exponential growth fit in Origin software.
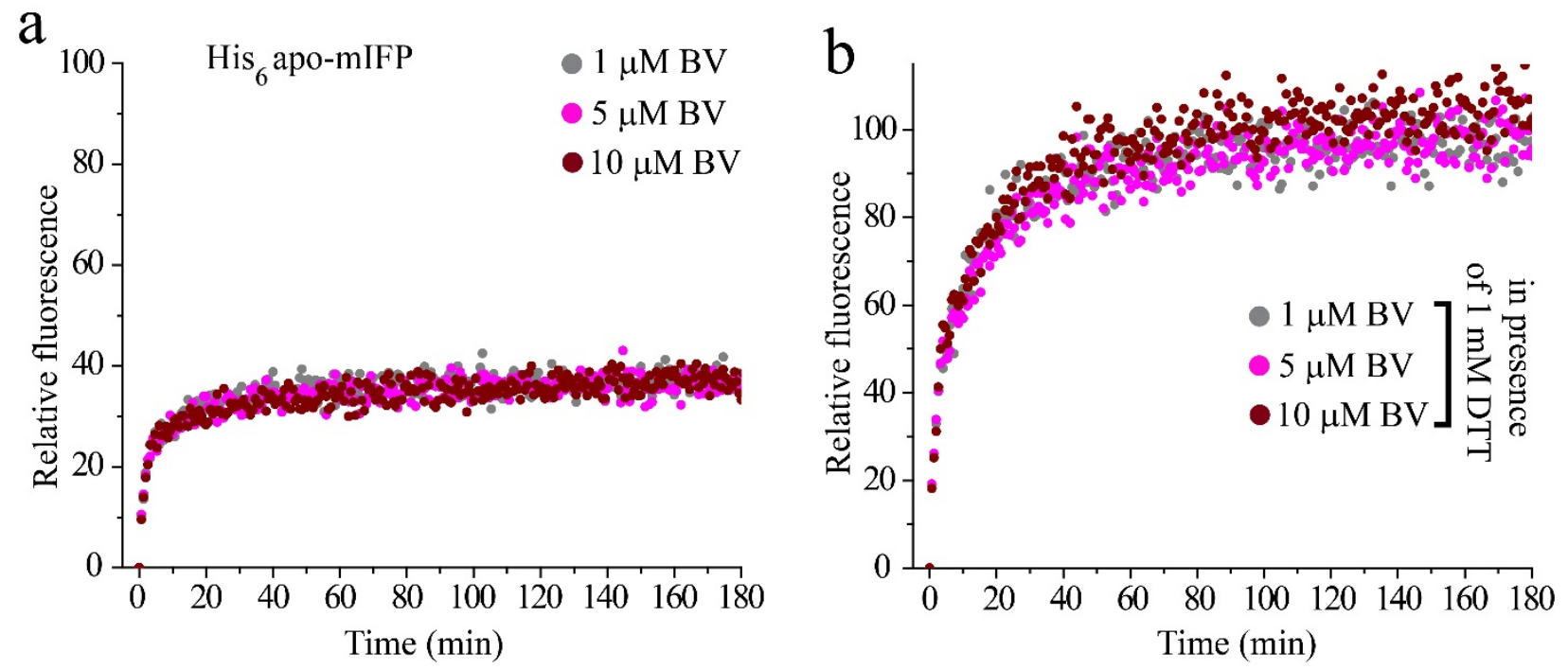

Figure S10. Maturation kinetics of apo-protein in presence of different concentrations of BV: (a) $\mathrm{His}_{6}$ apo-mIFP and (c) $\mathrm{His}_{6}$ apo-mIFP in presence of $1 \mathrm{mM}$ DTT. 


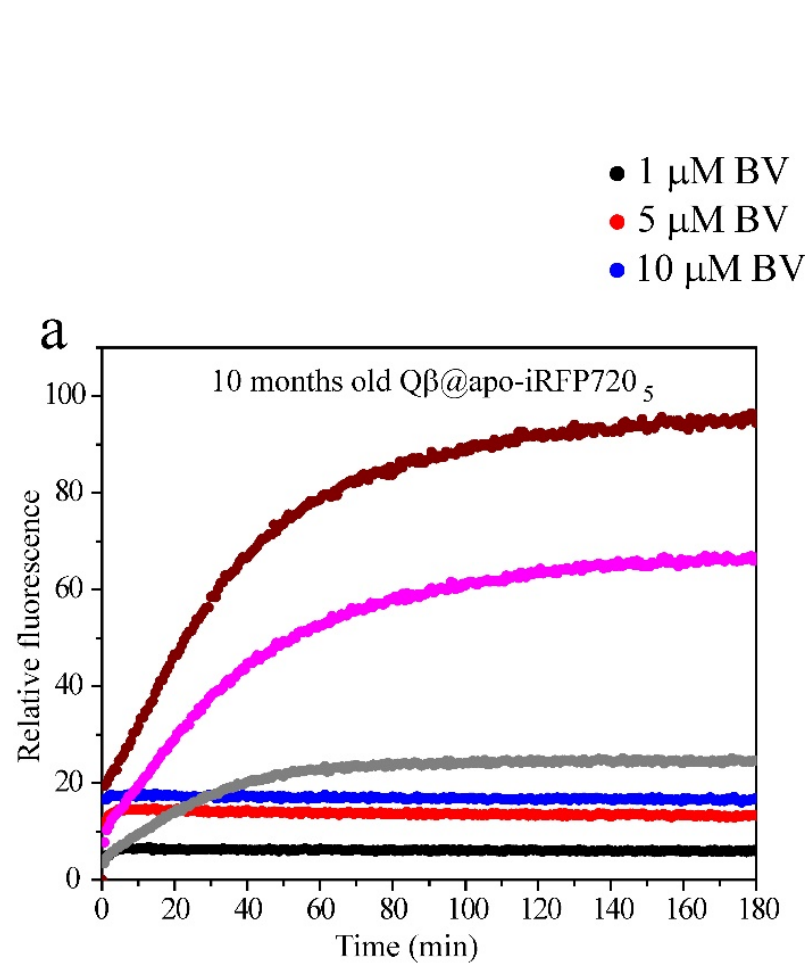

in presence

of $1 \mathrm{mM}$ DTT
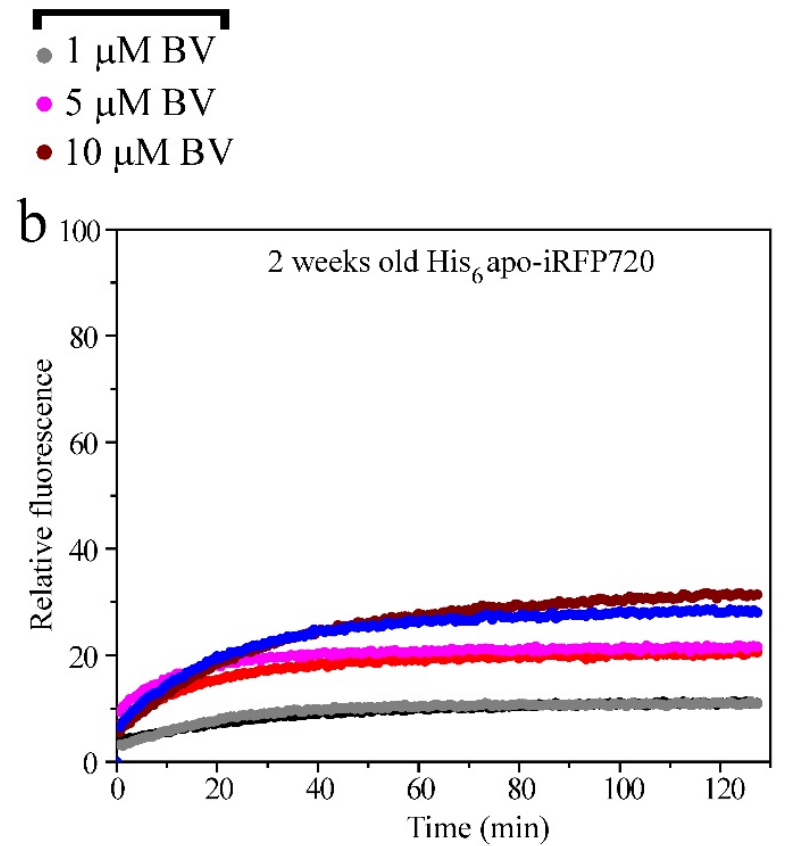

Figure S11. Maturation kinetics of old apo-protein in presence of different concentrations of BV with or without 1 mM DTT (a) Qß@apo-iRFP720 and (b) His apo-iRFP720.
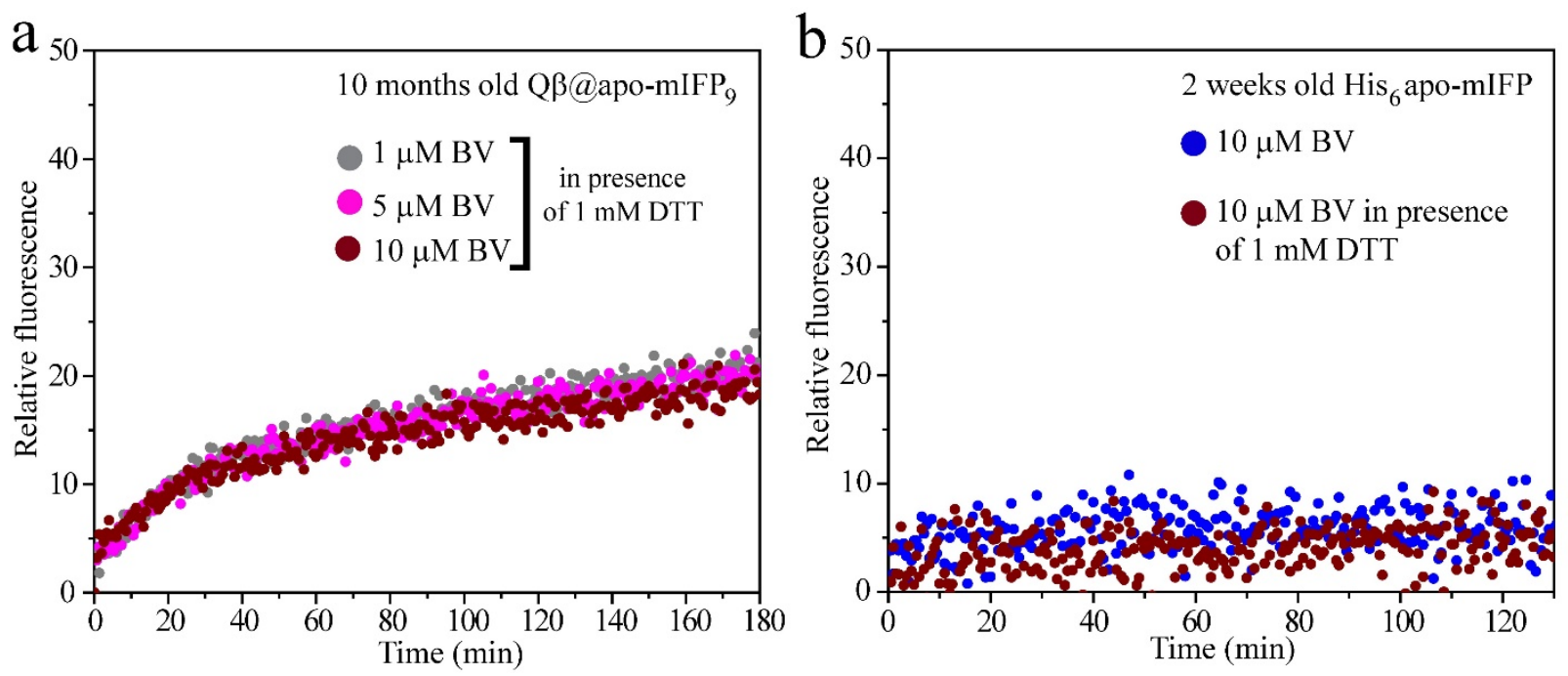

Figure S12. Maturation kinetics of old apo-protein in presence of different concentrations of BV with or without 1 mM DTT (a) Q $\beta @$ apo-mIFP 9 and (b) His ${ }_{6}$ apo-mIFP. 
Figure S13. Effect of DTT+BV treatment on partially mature DP-Qß@ mIFP $_{8}$ particles (200 nM particles, $1 \mu \mathrm{M}$ mIFP, $1 \mu \mathrm{M}$ $\mathrm{BV}, 1 \mu \mathrm{M}$ DTT, RT, overnight).

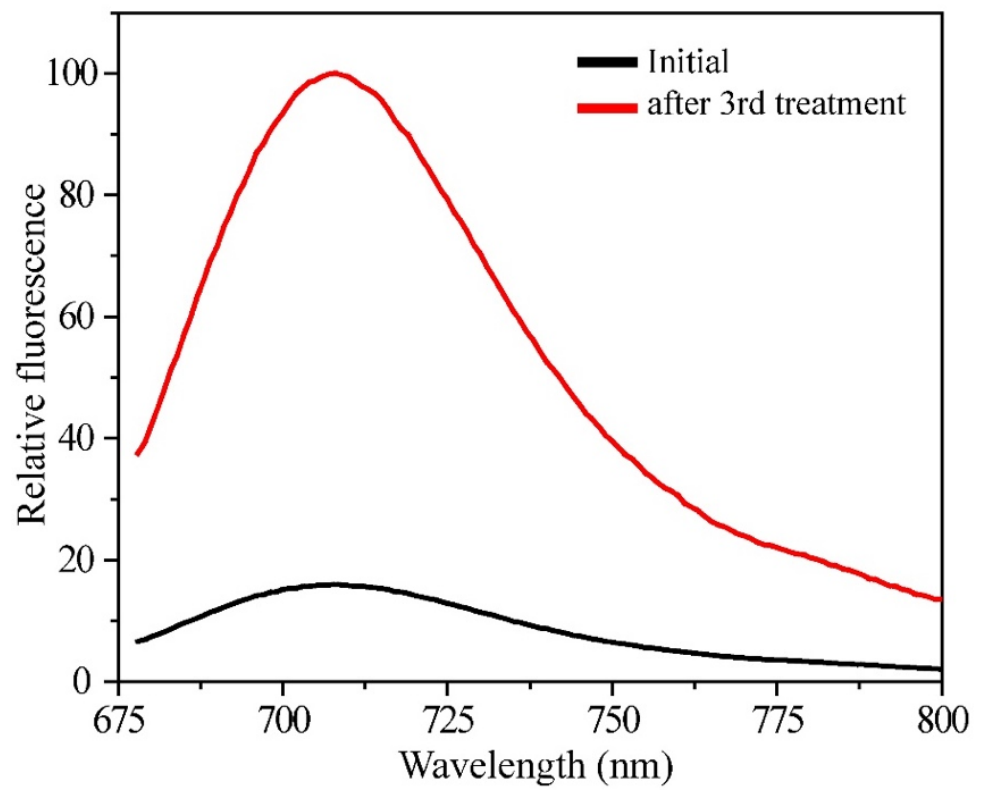

